\title{
CURVAS DIFERENCIADAS DE ÍNDICE DE SITIO PARA Vochysia guatemalensis EN LA REGIÓN HUETAR NORTE Y HUETAR CARIBE DE COSTA RICA
}

\author{
Fernando Mora ${ }^{1 / *}$, Víctor Meza $a^{* *}$, Ángel Porras ${ }^{* * *}$, Carlos Sandï $^{* * * *}$, Luis Aguilar $^{* * * * * *}$ \\ Palabras clave: Vochysia guatemalensis; crecimiento en altura dominante; calidad de sitio; índice de sitio; \\ curvas diferenciadas; Costa Rica. \\ Keywords: Vochysia guatemalensis; dominant height growth; site quality; site index; \\ differentiated curves; Costa Rica.
}

Recibido: $18 / 05 / 18$

Aceptado: 18/01/19

RESUMEN

El análisis del crecimiento en altura dominante para Vochysia guatemalensis, basado en una muestra de parcelas permanentes localizadas en las regiones Huetar Norte y Huetar Caribe, permitió distinguir 2 patrones de crecimiento disímiles y a la vez desarrollar curvas diferenciadas de índice de sitio. Para llevar a cabo este trabajo, se utilizó toda la información disponible sobre el crecimiento de esta especie, proveniente de organizaciones públicas y privadas, lo cual da origen a una muestra de 43 parcelas permanentes de muestreo; las mismas produjeron 397 promedios de altura dominante-edad con los que se ajustaron todos los modelos. El primer paso fue clasificar el crecimiento en altura dominante y como resultado se crearon, preliminarmente, 3 clases de calidad de sitio; esto permitió comprobar la existencia de 2 diferentes patrones de crecimiento en la muestra: uno representado por las clases I y II (Muestra 1) y el otro por la clase III (Muestra 2). La prueba de Levene no rechazó

\footnotetext{
1 Autor para correspondencia. Correo electrónico: fernando.mora.chacon@una.cr

* Investigador jubilado, Costa Rica. (D) 0000-0002-3910-2413.

** Universidad Nacional, Instituto de Investigación y Servicios Forestales, Costa Rica. (iD) 0000-0002-8223-4761.
}

\begin{tabular}{|c|c|}
\hline & ABSTRACT \\
\hline $\begin{array}{l}\text { Voch } \\
\text { Caril } \\
\text { The : } \\
\text { Voch } \\
\text { perm } \\
\text { and } \\
\text { Rica, } \\
\text { patter } \\
\text { curve } \\
\text { infori } \\
\text { speci } \\
\text { was u } \\
\text { samp } \\
\text { heigh } \\
\text { were } \\
\text { the d } \\
\text { site c } \\
\text { it all } \\
\text { grow } \\
\text { by cl }\end{array}$ & $\begin{array}{l}\text { Differentiated site index curves for } \\
\text { sia guatemalensis in the Northern and } \\
\text { bean Huetar Regions of Costa Rica. } \\
\text { nalysis of the dominant height growth for } \\
\text { sia guatemalensis, based on a sample of } \\
\text { anent plots located in the Northern Huetar } \\
\text { he Caribbean Huetar regions of Costa } \\
\text { allowed to distinguish } 2 \text { dissimilar growth } \\
\text { ns and develop differentiated site index } \\
\text { s. In order to carry out this work, all the } \\
\text { nation available about the growth of this } \\
\text { s from public and private organizations } \\
\text { sed, giving rise to a sample of } 43 \text { permanent } \\
\text { ing plots; which produced } 397 \text { dominant } \\
\text { t-age averages, with which all models } \\
\text { adjusted. The first step was to classify } \\
\text { ominant height growth and as a result } 3 \\
\text { uality classes were preliminarily created; } \\
\text { owed proving the existence of } 2 \text { different } \\
\text { h patterns in the sample: one represented } \\
\text { isses I and II (sample } 1 \text { ), and the other by }\end{array}$ \\
\hline & $\begin{array}{l}\text { Universidad Nacional, Instituto de Estudios Sociales } \\
\text { en Población, Costa Rica. } \\
\text { (D) } 0000-0002-9394-1912 \text {. }\end{array}$ \\
\hline & $\begin{array}{l}\text { Universidad EARTH, Costa Rica, Finca Forestal } \\
\text { Académica. (D) 0000-0002-6385-8499. }\end{array}$ \\
\hline$* * * * *$ & $\begin{array}{l}\text { Consultor privado, Costa Rica. } \\
\text { (DD) } 0000-0002-0997-5483 \text {. }\end{array}$ \\
\hline
\end{tabular}


la hipótesis de igualdad de varianzas entre las muestras, lo cual indica que existe una alta probabilidad de que las muestras 1 y 2 provengan de la misma población, mientras que la prueba $t$ rechazó la hipótesis nula de igualdad de medias, al revelar que existen diferencias significativas en el promedio de las alturas dominantes de ambas muestras. Debido a esto, se decidió ajustar 2 juegos de curvas separadas de índice de sitio, con base en las discrepancias observadas entre las muestras. Para construir las curvas se utilizó una edad base de 9 años. Para ambas muestras se probaron 8 funciones lineales y no lineales (pero linealizables) y 12 modelos no lineales. Para la Muestra 1 la función seleccionada fue la de Bailey y Clutter, y para la Muestra 2 se utilizó la función de Prodan; ambas ecuaciones presentaron un buen ajuste estadístico y mostraron un buen comportamiento cuando se graficaron contra los valores observados. La ecuación para la Muestra 1 presentó los siguientes estadísticos $\mathrm{r}=-0,94$; $\mathrm{R}^{2} \mathrm{adj}=0,89 ; \mathrm{MSE}=0,0565 ; \mathrm{SEE}=0,2378$; y la ecuación para la Muestra 2 produjo los siguientes resultados: $r=0,93 ; R^{2}$ adj $=0,87 ; \mathrm{MSE}=0,3886$; $\mathrm{SEE}=0,6234$.

\section{INTRODUCCIÓN}

Vochysia guatemalensis (Donn. Smith) es una de las principales especies forestales nativas utilizadas en la reforestación comercial en Costa Rica. Para el 2004, el área plantada en la región Huetar Norte, alcanzó el 25\% del total reforestado en todo el país con esta especie (Solís y Moya 2004), lo cual correspondía a 947 hectáreas; 10 años más tarde en el 2014 alcanzó 2116 hectáreas, que corresponde a un 64\% del área plantada con esta especie en todo el país (INEC 2014). Debido a su facultad de acumular aluminio en el follaje, así como en otros tejidos del árbol (Chenery y Sporne 1976, Foy et al. 1978, citados por Pérez 1993), se le considera una especie hyperacumuladora o class III (sample 2). Levene test did not reject the hypothesis of equality of variances between the samples, which indicates there is a high probability that Samples 1 and 2 may come from the same population, while $t$ test rejected the null hypothesis of equality of means, revealing there are significant differences in the average of the dominant heights of both samples. Because of this, it was decided to adjust 2 separated sets of site index curves, based on the observed discrepancies. To build the curves, a base age of 9 years were used. In both cases, eight linear and non-linear (but linearizable) functions and 12 non-linear models were tested. For sample 1 the selected function was that of Bailey and Clutter, and for sample 2 the selected function was that of Prodan; both equations showed a good statistical fit and a good performance when graphed against the observed values. The equation for sample 1, presented the following statistics: $\mathrm{r}=-0.94$; $\mathrm{R}^{2} \mathrm{adj}=0.89 ; \mathrm{MSE}=0.0565 ; \mathrm{SEE}=0.2378$; and the second equation for sample 2 , showed the following results: $\mathrm{r}=0.93 ; \mathrm{R}^{2} \mathrm{adj}=0.87$; $\mathrm{MSE}=$ $0.3886 ;$ SEE $=0.6234$. tolerante al aluminio (Camacho et al. 2017). Por esta cualidad y por su capacidad para ocupar terrenos con pastos degradados (Montagnini y Sancho 1994), se le reconoce un gran potencial para crecer mejor que otras especies forestales en los suelos ácidos y poco fértiles de la región tropical. Camacho et al. (2016) afirman que los árboles de esta especie tienen una ventaja competitiva, por encima de Tectona grandis y Gmelina arborea, debido a su mejor adaptabilidad a suelos bajos en $\mathrm{pH}$ y altos en aluminio. Por otro lado, $V$. guatemalensis presenta mayores tasas de crecimiento y productividad cuando se le compara con otras especies forestales nativas del país (Delgado 2002, Arias et al. 2011). Según lo anterior, ha dado lugar para que sea propuesta como una 
especie complementaria a las especies exóticas que dominan el mercado en los programas de reforestación locales.

\section{Crecimiento en altura y clasificación de sitios}

Los estudios para evaluar el desarrollo en altura de las especies forestales nativas han alcanzado en los últimos años, mayor importancia práctica, debido a su relación directa con el cálculo del volumen total de madera (Andrade 2002). En el caso de V. guatemalensis los pocos estudios que existen por lo general, han reportado alturas hasta edades muy tempranas, como por ejemplo, el estudio presentado por ACEN (1992), en donde se hace referencia a los datos de crecimiento en altura del proyecto de Ensayos Forestales (TRIAL) desarrollado por la OET. Esta deficiencia en la información limita las proyecciones del volumen para esta especie a edades más avanzadas (Arias et al. 2011).

En Costa Rica, los trabajos sobre calidad de sitio y clasificación de sitios para $V$. guatemalensis son muy escasos. De hecho, solamente se conocen unos pocos estudios publicados, uno de ellos realizado por Barraza y Díaz (1999), quienes de manera preliminar clasificaron 23 localidades en las cuales se encontraba creciendo la especie. Para construir el sistema de clasificación de sitios los autores definieron una edad base de 11 años y establecieron 3 clases de calidad de sitio: Baja (IS entre 24 y 27), Media (IS entre 27 y 33) y Alta (IS entre 33 y 36). Por su parte, COSEFORMA (2001) publicó una guía sobre V. Guatemalensis, en la cual se presenta un esquema para la clasificación de las plantaciones a través de la estratificación de las tasas de crecimiento diamétrico, lo cual permitió construir 3 escenarios de crecimiento en diámetro para esta especie. No obstante, este esquema de clasificación, basado en el crecimiento diamétrico, tiene su mayor aplicación en la definición de propuestas para el manejo silvícola de esta especie, más que para una clasificación de sitios.

Más recientemente, Mora et al. (2015) utilizaron una clasificación de sitios preliminar para evaluar los factores edáficos que influyen en el crecimiento de esta especie en la región Huetar Norte de Costa Rica. En la presente investigación, se utilizaron variables físicas y químicas de 2 tipos de suelos (Inceptisoles y Ultisoles), con el fin de explicar el comportamiento del índice de sitio (IS) y su dependencia de aquellas variables edáficas que mostraron algún tipo de relación con la calidad de los terrenos (fuera esta positiva o negativa) para $V$. guatemalensis.

Se propuso evaluar los patrones de crecimiento de la altura dominante y desarrollar un sistema para clasificar la calidad de los terrenos plantados con $V$. guatemalensis, al utilizar el crecimiento de la altura dominante como un indicador de la calidad de sitio. Esta última constituye un concepto integrador, que resume las variadas condiciones que brindan los distintos lugares para el crecimiento de los árboles (Prodan et al. 1997). Bajo este punto de vista, la medida de la calidad de sitio refleja el potencial de productividad o capacidad productiva de un sitio, por lo que se convierte en una expresión de la capacidad de un área de terreno para producir madera o cualquier otro tipo de vegetación (Davis y Johnson 1987).

El objetivo del presente trabajo fue seleccionar un modelo adecuado, de acuerdo con los criterios señalados por Curtis (1964) y Bailey y Clutter (1974), Mora et al. (2014), que represente acertadamente el crecimiento de la altura dominante para Vochysia guatemalensis y que permita construir curvas actualizadas de índice de sitio (IS), con el fin de facilitar la toma de decisiones relacionadas con el manejo silvicultural de esta especie, en la regiones Huetar Norte y Huetar Caribe de Costa Rica.

\section{MATERIALES Y MÉTODOS}

\section{Área de estudio}

Para llevar a cabo este trabajo fue necesario compilar la información existente acerca del crecimiento de $V$. guatemalensis, proveniente de diferentes organizaciones públicas y privadas, como FUNDECOR, EARTH, COSEFORMA y TEC. El área de estudio abarca las regiones 
Huetar Norte y Huetar Caribe de Costa Rica, donde las Parcelas Permanentes de Muestreo (PPM) de COSEFORMA-TEC se distribuyen principalmente en los cantones de Los Chiles y
San Carlos; las de FUNDECOR se concentran en los cantones de Sarapiquí y Pocosí; y las de la EARTH se ubican en los cantones de Guácimo y Siquirres (Figura 1).

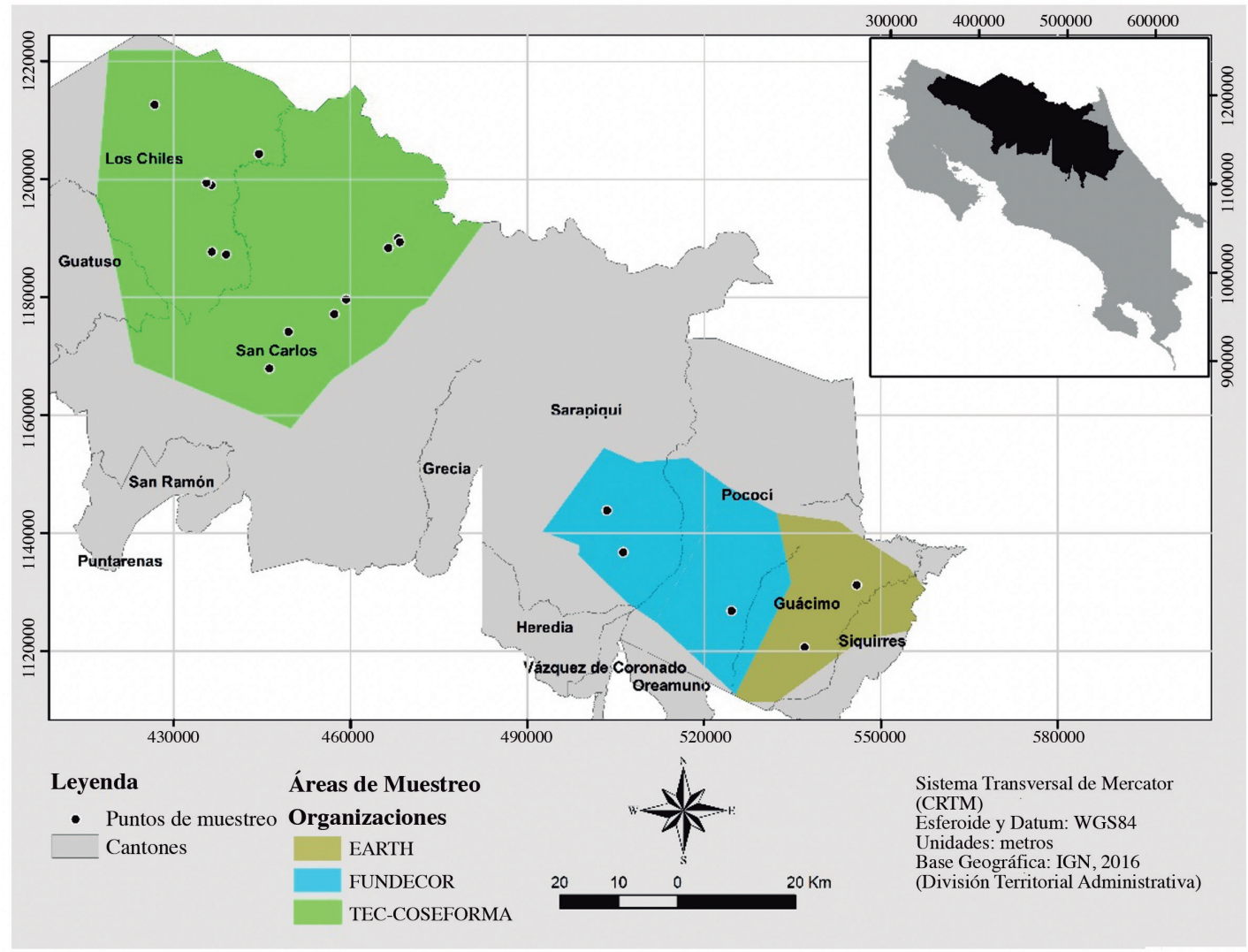

Fig. 1. Distribución de las parcelas permanentes y el área de cobertura del estudio para cada organización involucrada, regiones Huetar Norte y Huetar Caribe, Costa Rica.

\section{Recopilación de los datos}

El proceso de compilación dio como resultado una base de datos compuesta por los promedios de 43 parcelas permanentes de muestreo (PPM), las cuales produjeron 397 pares de datos altura dominante-edad. El área total del estudio abarca $4233 \mathrm{~km}^{2}$ (Cuadro 1). 
Cuadro 1. Descripción de la muestra y de las áreas de estudio en las regiones Huetar Norte y Huetar Caribe, Costa Rica.

\begin{tabular}{lccccc}
\hline Organización & $\begin{array}{c}\mathrm{N}^{\mathrm{o}} \text { de } \\
\text { parcelas }\end{array}$ & $\begin{array}{c}\text { Tamaño de } \\
\text { parcelas } \\
\left(\mathrm{m}^{2}\right)\end{array}$ & $\begin{array}{c}\text { Altitud } \\
(\mathrm{msnm})\end{array}$ & $\begin{array}{c}\text { Precipitación } \\
(\mathrm{mm})\end{array}$ & $\begin{array}{c}\text { Área de } \\
\text { cobertura } \\
\left(\mathrm{km}^{2}\right)\end{array}$ \\
\hline COSEFORMA-TEC & 12 & 400 & $70-212$ & $2200-3200$ & 2750 \\
FUNDECOR & 14 & 500 & $30-400$ & $3000-5000$ & 958 \\
UNIVERSIDAD EARTH & 17 & 600 & 64 & 3464 & 525 \\
\hline
\end{tabular}

La mayoría de las parcelas fueron medidas desde 1 año hasta 16,5 años (esta edad se considera adecuada, ya que los turnos de corta para esta especie no superan los 12 años). En todas las parcelas se registró la edad para cada medición, se midió el diámetro (Dap) en centímetros y la altura total (Htot) en metros; además, se calculó la altura dominante como el promedio en altura total de los árboles más altos en un número equivalente al $10 \%$ de los árboles de la parcela.

Por tratarse de una base de datos construida a partir del trabajo de 4 instituciones, fue necesario llevar a cabo una revisión y depuración de la misma en busca de inconsistencias. Se trató de corregirlas en todos los casos, cuando esto fue posible, y se removieron de la base de datos cuando no se pudo aplicar la corrección deseada.

\section{Identificación de los patrones de crecimiento mostrados por la altura dominante}

Con el propósito de encontrar evidencia empírica de la existencia de clases de calidad de sitio en las localidades, donde se encuentra creciendo $V$. guatemalensis, fue necesario seguir los siguientes pasos:

1. Se graficaron los datos de altura dominante en metros (definida como el promedio en altura total del $10 \%$ de los árboles más altos de la parcela) versus la edad en años, con el fin de estudiar su comportamiento y detectar las diferencias existentes entre las parcelas a la edad de 8 años. Esto permitió identificar, de manera preliminar, 3 clases de calidad de sitio basados en el crecimiento de la altura dominante a la edad mencionada. Esta clasificación constituye un paso preliminar hacia la estratificación definitiva de la muestra.

2. Posteriormente, se graficaron los datos de las 3 clases por separado, para realizar el análisis del crecimiento en altura y estudiar el comportamiento de varios modelos lineales y no lineales con cada uno de los grupos. El proceso permitió detectar la existencia de 2 patrones de crecimiento (2 maneras de crecer) para V.guatemalensis.

3. Se procedió a conformar un primer grupo con las clases I y II (llamada Muestra 1), ya que mostraron un comportamiento muy similar. El segundo grupo quedó conformado por los datos de la clase III (Muestra 2), los cuales difieren en su comportamiento con respecto a los datos de la Muestra 1.

4. Durante esta fase, se optó por cambiar la edad de referencia de 8 a 9 años, debido a que esta última estaba bien representada en los datos, mostraba una desviación estándar menor y se encontraba más próxima al turno de rotación.

5. Para determinar si ambos grupos o muestras provienen de una misma población, o si representan poblaciones diferentes, se aplicó la prueba inferencial de Levene (1960).

6. Posteriormente, para dilucidar si ambas muestras son o no estadísticamente 
diferentes, se aplicó la prueba t para la igualdad de medias.

7. Con el fin de poner en evidencia las discrepancias encontradas para ambas muestras, se procedió a crear un gráfico altura dominante-edad que muestra las diferencias en el comportamiento de ambos grupos de datos.

8. Para apoyar la teoría estadística, se aportó evidencia empírica al graficar el incremento corriente anual (ICA) y el incremento medio anual (IMA) de la altura dominante contra la edad.

9. Finalmente, ante la imposibilidad de encontrar un modelo lo suficientemente flexible para representar ambos patrones de crecimiento, se resolvió trabajar con cada grupo por separado y utilizar un modelo diferente para ajustar las curvas de índice de sitio, utilizando un enfoque polimórfico en ambos casos. Los 2 juegos de curvas se representan en un mismo gráfico, con el fin de contrastar la forma de las curvas y las tendencias que ambas muestras representan.

\section{Selección del modelo de mejor ajuste para la construcción de las curvas de índice de sitio}

Para cada grupo de datos se probaron los mismos modelos de regresión, lo cual permitió seleccionar el modelo que mejor ajustó los datos, tanto para la Muestra 1 como para la Muestra 2. Para evaluar cada modelo se tomaron en cuenta los siguientes estadísticos: $r=$ coeficiente de correlación; $\mathrm{R}^{2}$ = coeficiente de determinación; $\mathrm{R}^{2} \mathrm{adj}=$ coeficiente de determinación ajustado; MSE = cuadrado medio del error; SEE $=$ error estándar de las estimaciones; y MAE = error medio absoluto. Además, se graficaron los valores estimados versus los valores observados, con el fin de evaluar gráficamente el ajuste de los datos.

En una primera fase, se trabajó con los siguientes modelos lineales: Logarítmico, Semilogarítmico, Cuadrático, Doble Recíproco, Raíz cuadrada de Y-Logaritmo de X y la ecuación de Schumacher (1939) en su forma lineal. También se probaron 2 modelos no lineales, pero linealizables: Prodan (1951), citado por Thompson et al. 1984, y Bailey y Clutter (1974), los cuales, a través de una serie de transformaciones, es posible ajustarlos mediante técnicas de cuadrados mínimos lineales.

En una segunda fase se trabajó con los siguientes modelos no lineales: Mistcherlich o Monomolecular, Weibull, Gompertz y ChapmanRichards. Todos estos modelos se pueden obtener a partir de una misma función, la ecuación de von Bertanlanffy también fue ensayada con los datos de la muestra. A esta lista se agregaron 6 modelos más, los cuales constituyen variantes de algunos de los modelos anteriores. A partir del modelo de Gompertz se obtiene el modelo Gompertz modificado; de la ecuación de Richards (1959) se obtienen el Modelo Natural de Crecimiento cuando $\mathrm{c}=1$ y la Función Logística Generalizada cuando $\mathrm{c}=-1$; de esta última se puede obtener la Función Logística o Autocatalítica cuando $d=1$. También se probaron 2 versiones de ambas funciones logísticas, reescritas y recompuestas por Sit y Poulin-Costello (1994). Finalmente, se incluyó el modelo de Schumacher (1939) en su forma no lineal.

\section{Desarrollo teórico-simbólico de los modelos utilizados para la construcción de las curvas de índice de sitio}

Debido a las discrepancias irreconciliables observadas en los patrones de crecimiento que presenta $V$. guatemalensis, en las regiones Huetar Norte y Huetar Caribe de Costa Rica, se procedió a ajustar modelos separados para cada tendencia. El modelo de Bailey y Clutter se ajustó para el grupo de parcelas que componen la Muestra 1 (clases I y II) y el modelo de Prodan se utilizó para los datos de la Muestra 2 (clase III). Con el fin de elevar el valor didáctico del actual trabajo, las funciones utilizadas y su desarrollo teórico-simbólico, se presentan a continuación. 


\section{Modelo de Bailey y Clutter}

Para los datos altura-edad correspondientes a las plantaciones de V. Guatemalensis representadas en la Muestra 1 (clases I y II), el modelo utilizado fue la versión generalizada del modelo de Schumacher (1939), modificado por Bailey y Clutter (1974). Su desarrollo simbólico se presenta a continuación:

$$
\operatorname{Ln}(\mathrm{H})=\mathrm{a}-\mathrm{b} *(1 / \mathrm{E})^{\wedge} \mathrm{c}
$$

Donde, $\mathbf{a}, \mathbf{b}$ y $\mathbf{c}$ son los coeficientes a ser ajustados $\mathbf{H}$ es la altura dominante (m), $\mathbf{E}$ representa la edad (en años) y $\mathbf{L n}$ es el logaritmo natural.

Este modelo, al igual que el de Schumacher, asume que: "el logaritmo de la altura dominante es una función lineal del recíproco de la edad", sin embargo, se diferencia en que este incorpora un tercer coeficiente $\mathbf{c}$, que actúa como exponente de la variable independiente. Esta condición le confiere varios atributos importantes: por un lado, se vuelve más flexible, al ser capaz de asumir formas diferentes, lo cual le permite ajustar datos de muy diversos orígenes; por otro lado, la forma de las curvas se mantiene aunque cambie la edad base (Bailey y Clutter 1974). En todo caso, según Alder (1980), para la mayoría de las especies, los valores apropiados de $\mathbf{c}$ varían entre 0,2 y 2,0 .

Es necesario aclarar que este modelo no lineal puede ser ajustado linealmente por medio de un proceso de 2 etapas (Bailey y Clutter 1974):

Etapa 1. Se ajusta la primera derivada de la ecuación de regresión para estimar $\mathbf{c}$. La derivada de esta ecuación es de la forma:

$$
\operatorname{Ln}(\mathrm{I} / \mathrm{H})=\operatorname{Ln}(-\mathrm{cb})+(\mathrm{c}+1) * \operatorname{Ln}(1 / \mathrm{E})
$$

Donde, Ln(-cb) corresponde al punto de intercepción y $(\mathbf{c}+\mathbf{1})$ equivale a la pendiente del modelo; I es el incremento corriente anual en altura dominante; $\mathbf{H}$ y $\mathbf{E}$ fueron previamente definidos. Como esta igualdad es lineal en sus parámetros, los procedimientos normales de regresión van a producir una estimación de $\hat{\mathbf{c}}+\mathbf{1}$, del cual $\hat{\mathbf{c}}$ puede ser derivado al restar el valor de 1 a la pendiente de esta ecuación (Bailey y Clutter 1974). Para aplicar este método se requiere información producida a partir de mediciones sucesivas realizadas en parcelas permanentes, o bien, por análisis fustal, pues es necesario contar con datos del incremento corriente anual en altura para cada una de las edades (Mora y Meza 2003).

Etapa 2. Se utiliza $\hat{\mathbf{c}}$ en la ecuación básica (1) y se ajustan los datos por medio de la regresión lineal simple,

$$
\operatorname{Ln}(H)=a-b *(1 / E)^{\wedge \hat{c}}
$$

Ahora bien, antes de construir las curvas de índice de sitio, es necesario recordar la definición del índice de sitio, la cual dice que: "cuando la edad (E) asume el valor de la edad base (Eb), la altura dominante $(\mathbf{H})$ es igual al índice de sitio (IS)". Al tener en cuenta este enunciado, se procedió a sustituir estas 2 variables en la ecuación anterior, por lo que quedó expresada de la siguiente forma,

$$
\operatorname{Ln}(\mathrm{IS})=\mathrm{a}-\mathrm{b} *(1 / \mathrm{Eb})^{\wedge \hat{c}}
$$

Para construir curvas polimórficas de índice de sitio es necesario poner a variar el parámetro b, al cual llamaremos "coeficiente de sitio" ( $\left.\mathbf{b}_{\mathbf{i}}\right)$, ya que este varía con el índice de sitio. De este modo, despejando $\mathbf{b}_{\mathbf{i}}$ en la ecuación (3), se tiene,

$$
-b_{i}=(a-\operatorname{Ln}(\mathrm{IS})) *(E b)^{\wedge \hat{c}}
$$

Sustituyendo en (1), se puede tener,

$$
\begin{aligned}
& \operatorname{Ln}(\mathrm{H})=\mathrm{a}+\left\{(\mathrm{a}-\operatorname{Ln}(\mathrm{IS})) *\left(\mathrm{~Eb}^{\wedge \hat{c}}\right)\right\} *\left(1 / \mathrm{E}_{\mathrm{i}}\right)^{\wedge \hat{c}} \\
& =\mathrm{a}+(\mathrm{a}-\operatorname{Ln}(\mathrm{IS})) *\left\{\mathrm{~Eb} / \mathrm{E}_{\mathrm{i}}\right\}^{\wedge \hat{c}}
\end{aligned}
$$

Se eliminan los logaritmos naturales (base e) y se obtiene,

$$
\mathrm{H}=\mathrm{e}^{\wedge \mathrm{a}} *\left[\mathrm{IS} / \mathrm{e}^{\wedge}\right]^{\wedge\{\mathrm{Eb} / \mathrm{Ei}\}^{\wedge} \hat{c}}
$$


Donde e es la base de los logaritmos naturales (e $=2,71828)$; IS es el índice de sitio a escoger; a y ĉ fueron definidos en la ecuación básica; $\mathbf{E}_{\mathbf{i}}$ asume todos los valores de la edad; y Eb es la edad base (o edad clave), que en este caso es igual a 9 años.

\section{Modelo de Prodan}

En el caso de los datos que componen la Muestra 2 (clase III), se utilizó el modelo de Prodan. El desarrollo simbólico del mismo se presenta a continuación:

$$
\mathrm{H}=\left(\mathrm{E}^{2}\right) /\left(\mathrm{a}+\mathrm{b} * \mathrm{E}+\mathrm{c} * \mathrm{E}^{2}\right)
$$

Donde $\mathbf{a}, \mathbf{b}$ y $\mathbf{c}$ son los coeficientes a ser ajustados; $\mathbf{H}$ es la altura dominante (m) y $\mathbf{E}$ representa la edad (en años). Para ajustar este modelo, mediante mínimos cuadrados, es necesario remover la variable $\mathbf{E}^{\mathbf{2}}$ del numerador de la fracción, de manera que al realizar el despeje esta variable pasa al lado contrario del igual,

$$
E^{2} / H=a+b * E+c * E^{2}
$$

Con el fin de facilitar la manipulación de esta función, se hace necesario crear una nueva variable, la cual llamaremos $\mathbf{Z}$; de forma tal que, $\mathbf{Z}=\left(\mathbf{E}^{2} / \mathbf{H}\right)$. Entonces,

$$
\mathrm{Z}=\mathrm{a}+\mathrm{b} * \mathrm{E}+\mathrm{c} * \mathrm{E}^{2}
$$

Tomando en cuenta la definición del índice de sitio, señalada en la página anterior, se sabe que cuando $\mathrm{E}=\mathrm{Eb}$, entonces $\mathrm{H}=\mathrm{IS}$. De esta manera, sustituyendo en (8), se tiene,

$$
\mathrm{Eb}^{2} / \mathrm{IS}=\mathrm{a}+\mathrm{b} * \mathrm{~Eb}+\mathrm{c} * \mathrm{~Eb}^{2}
$$

Para construir curvas polimórficas con este modelo b pasa a depender del índice de sitio, por eso se denomina "coeficiente de sitio" $\left(\mathbf{b}_{\mathbf{i}}\right)$ y el mismo puede ser despejado de la ecuación anterior de la siguiente manera,

$$
b_{i}=\{(E b / I S)-(a / E b)-c * E b\}
$$

Al sustituir $\mathbf{b}_{\mathbf{i}}$ en la fórmula (7) se obtiene la siguiente función,

$$
\begin{aligned}
& \mathrm{H}=\mathrm{E}_{\mathrm{i}}^{2} /(\mathrm{a}+\{(\mathrm{Eb} / \mathrm{IS})-(\mathrm{a} / \mathrm{Eb})-(\mathrm{c} * \mathrm{~Eb})\} * \\
& \left.\mathrm{E}_{\mathrm{i}}+\left(\mathrm{c} * \mathrm{E}_{\mathrm{i}}^{2}\right)\right)
\end{aligned}
$$

Donde a y c son los mismos coeficientes de la ecuación original; $\mathbf{E}_{\mathbf{i}}$ es cualquier edad; y Eb es la edad clave (o edad base), que para fines del presente estudio es igual a 9 años.

\section{RESULTADOS Y DISCUSIÓN}

\section{Definición de las categorías de crecimiento en altura dominante}

El análisis de los datos permitió identificar 2 patrones de crecimiento claramente diferenciados; esto por cuanto los datos definen $2 \mathrm{~s}$ zonas en el gráfico (Figura 2). En la franja superior se encuentran los valores que representan los mejores sitios y corresponden a la Muestra 1 (clases I y II), y en la banda inferior se localizan las parcelas de peor crecimiento, correspondientes a la Muestra 2 (clase III). Mientras que los datos de la Muestra 1 presentan una mayor pendiente y dan muestras de un crecimiento sostenido, los datos de la Muestra 2 presentan una pendiente más leve y una tendencia a buscar la horizontalidad a una edad muy temprana; esto sugiere que pronto podrían desacelerar aun más su lento crecimiento. 


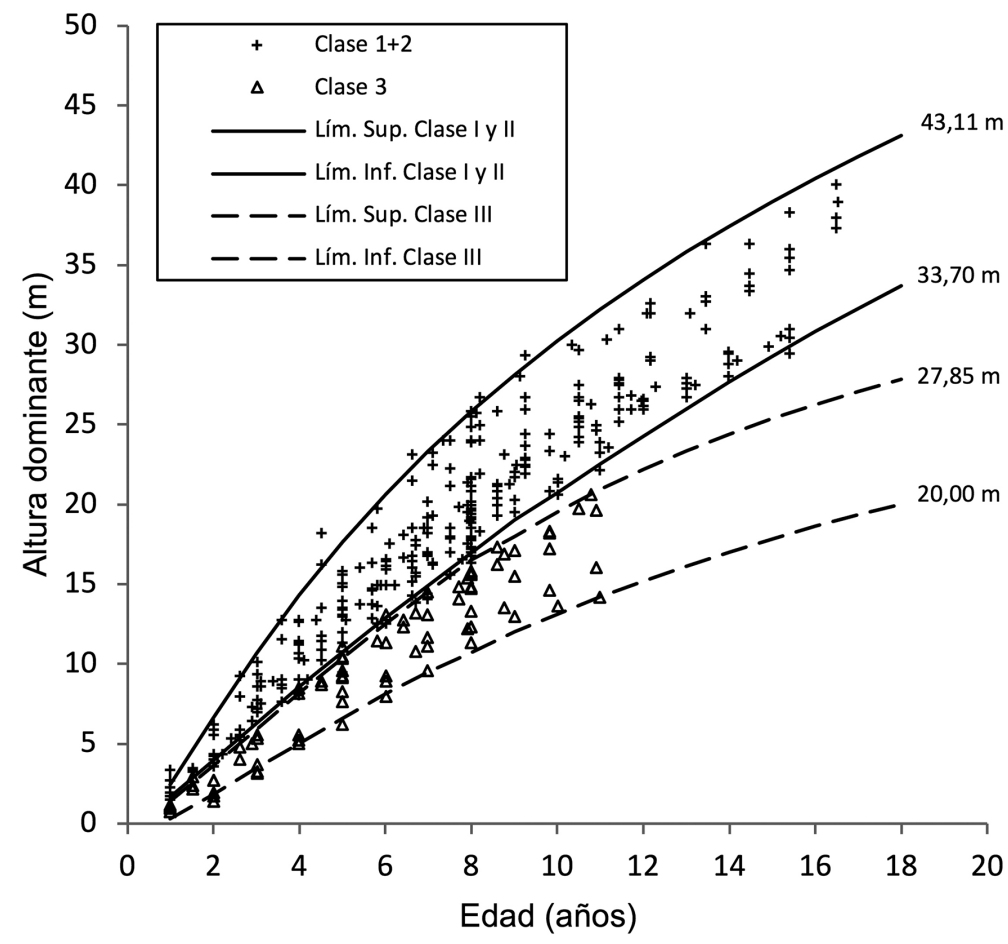

Fig. 2. Dispersión de los datos que muestran las discrepancias observadas en el patrón de crecimiento de la altura dominante para Vochysia guatemalensis, en las regiones Huetar Norte y Huetar Caribe de Costa Rica.

En las primeras edades no se distingue con claridad un patrón diferenciado en la forma de crecer de $V$. guatemalensis, pero después de la edad de 8 años empieza a visualizarse una tendencia más clara en los promedios de la Muestra 2 (clase III), los cuales comienzan a separarse de la masa de datos que conforman la Muestra 1 (clases I y II). A partir de esa edad se observa que las parcelas de la Muestra 2 comienzan a mostrar una reducción en la pendiente y la evidencia sugiere que muy pronto podrían dejar de crecer y hasta podrían alcanzar su asíntota a una edad muy temprana. Los sitios donde se encuentran creciendo las parcelas de la Muestra 1, se pueden considerar de muy buena calidad para esta especie, mientras que los sitios que corresponden a la Muestra 2 se pueden considerar como malos o muy malos.
Bajo este esquema, a la edad base de 9 años la Muestra 1 está delimitada entre las alturas $19,0 \mathrm{~m}$ y $28,13 \mathrm{~m}$; la Muestra 2, que representa a los sitios de peor calidad, queda definida entre las alturas $12,0 \mathrm{~m}$ y $18,0 \mathrm{~m}$.

Como se puede ver en la Figura 2, a la edad de referencia, la separación entre los 2 grupos de datos es muy leve, ya que la brecha entre ambas muestras se reduce a solamente un metro de distancia. Sin embargo, a la edad de 18 años el panorama cambia. La curva que representa el límite inferior de la Muestra 2 cierra con una altura de 20,0 m y la curva del límite superior de termina en $27,85 \mathrm{~m}$ a la edad señalada. Por otro lado, la curva que representa el límite inferior de la Muestra 1 cierra con una altura de $33,70 \mathrm{~m}$ a la edad de 18 años, mientras que la curva que actúa como límite superior alcanza una altura 
de 43,11 $\mathrm{m}$ a dicha edad. De esta manera, entre el límite inferior de la Muestra 1 y el límite superior de la Muestra 2, existe una brecha de casi $6,0 \mathrm{~m}$ de diferencia $(5,85 \mathrm{~m})$ a la edad de 18 años, lo que confirma el crecimiento diferenciado para ambas muestras.

\section{Teoría estadística que respalda las diferencias encontradas en la muestra}

\section{Prueba de Levene para la igualdad de varianzas}

Al aplicar la prueba de Levene se pudo determinar que esta no rechazó la hipótesis de igualdad de varianzas, lo cual indica con una alta probabilidad que las muestras proceden de una misma población. Como se puede ver en el Cuadro 2, el p-valor de 0,633 es mayor que el nivel de significancia de 0,05 , escogido para trabajar en este caso; lo que indica que no se puede rechazar la hipótesis nula.
Cuadro 2. Resultados de la aplicación de la prueba de Levene, utilizada para probar la hipótesis nula de igualdad de varianzas, para $V$. guatemalensis en las regiones Huetar Norte y Huetar Caribe de Costa Rica.

\begin{tabular}{|c|c|c|c|}
\hline \multirow[t]{2}{*}{ Variable } & \multirow[t]{2}{*}{ Supuestos } & \multicolumn{2}{|c|}{$\begin{array}{c}\text { Prueba de Levene } \\
\text { de calidad de } \\
\text { varianzas }\end{array}$} \\
\hline & & F & Sig. \\
\hline Altura & $\begin{array}{c}\text { Se asumen varianzas } \\
\text { iguales }\end{array}$ & 0,233 & 0,633 \\
\hline (m) & $\begin{array}{c}\text { No se asumen } \\
\text { varianzas iguales }\end{array}$ & & \\
\hline
\end{tabular}

\section{Prueba t para la igualdad de medias}

La prueba t rechazó la hipótesis nula de igualdad de medias, ya que el p-valor (Sig. bilateral) de 0,000 es menor al nivel de significancia de 0,05 , elegido para trabajar con estos datos (Cuadro 3). Además, el intervalo de confianza no incluyó el valor de cero, lo cual es una razón más para desechar la hipótesis nula. La evidencia indica que realmente existen diferencias significativas en el promedio de las alturas dominantes para ambas muestras.

Cuadro 3. Resultados de la aplicación de la prueba t, utilizada para probar la hipótesis nula de igualdad de medias, para $V$. guatemalensis en las regiones Huetar Norte y Huetar Caribe de Costa Rica.

\begin{tabular}{|c|c|c|c|c|c|c|}
\hline \multirow[b]{2}{*}{$\mathrm{t}$} & \multirow[b]{2}{*}{$\mathrm{gl}$} & \multicolumn{3}{|c|}{ Prueba t para la igualdad de medias } & \multicolumn{2}{|c|}{$\begin{array}{c}95 \% \text { de intervalo de confianza } \\
\text { de la diferencia }\end{array}$} \\
\hline & & Sig. (bilateral) & $\begin{array}{l}\text { Diferencia de } \\
\text { medias }\end{array}$ & $\begin{array}{l}\text { Diferencia de } \\
\text { error estándar }\end{array}$ & Inferior & Superior \\
\hline 8,139 & 28 & 0,000 & 6,8260 & 0,8387 & 5,10801 & 8,54399 \\
\hline 8,139 & 27,98 & 0,000 & 6,8260 & 0,8387 & 5,10795 & 8,54405 \\
\hline
\end{tabular}

\section{Representación de los patrones de crecimiento de la especie}

Para sustentar los resultados estadísticos obtenidos a través de la prueba de Levene y de la prueba t, se analizó el comportamiento de la variable altura dominante, en su desarrollo a través del tiempo, para las 2 muestras. Para esto se procedió a graficar el incremento corriente anual (ICA) y el incremento medio anual (IMA) de la altura dominante versus la edad. 
La Figura 3 presenta la relación entre el Incremento Corriente Anual (ICA) y la Edad para $V$.guatemalensis al crecer en 2 calidades de sitio. En estas curvas se manifestaron diferencias atribuibles a la capacidad productiva de los terrenos. En el mejor sitio, la curva de la Muestra 1 inició desde una posición superior cercana a los 3,0 m, por lo que alcanzó un ICAmáx de 2,77 m a la edad de 3 años. Por su parte, la curva de la Muestra 2 arranca lentamente, con un ICA de 1,16 m y solamente logra alcanzar un ICAmáx de 1,98 $\mathrm{m}$ a la edad de 6 años.

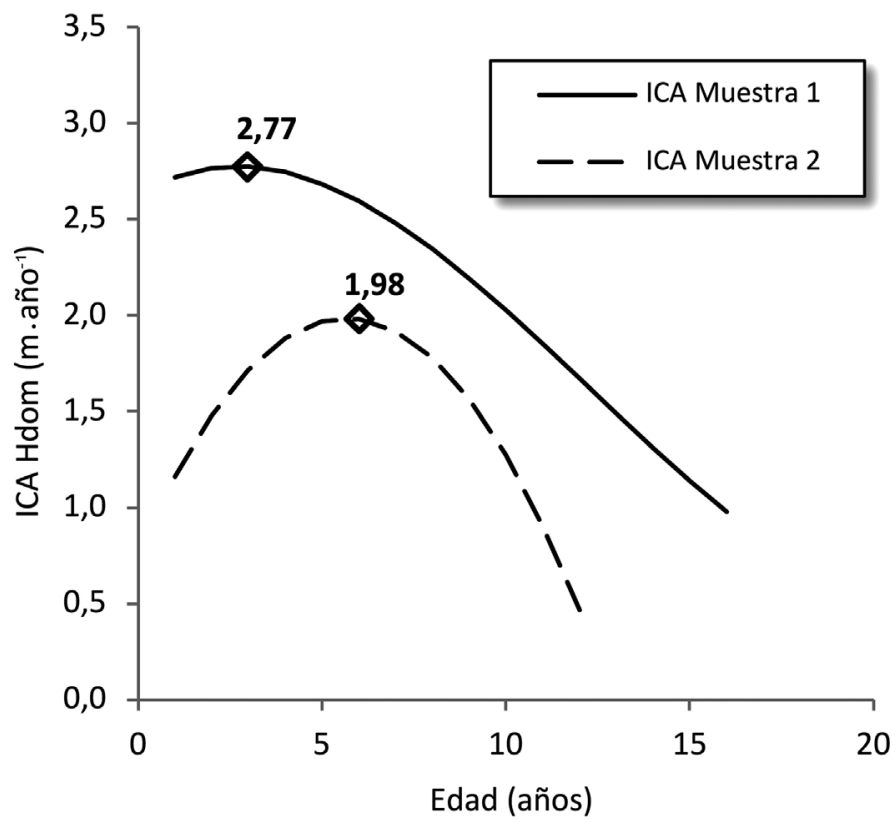

Fig. 3. Relación ICA(Hdom)-Edad para las muestras 1 y 2 de Vochysia guatemalensis, región Huetar Norte y Huetar Caribe, Costa Rica.

Las curvas representadas en la Figura 4 muestran la relación que existe entre el Incremento Medio Anual (IMA) y la Edad, para V. guatemalensis en 2 condiciones de calidad de sitio. Se puede ver claramente la posición superior que mantiene la primera curva, perteneciente a la Muestra 1, a lo largo de todo el recorrido sobre la segunda curva perteneciente a la Muestra 2. La primera presenta una forma más aplanada, alcanzando el IMA máximo a la edad de seis años; por su parte, la segunda curva no solo se aparta de la forma mostrada por la primera, sino que alcanza su IMA máximo a la edad de 8 años. 


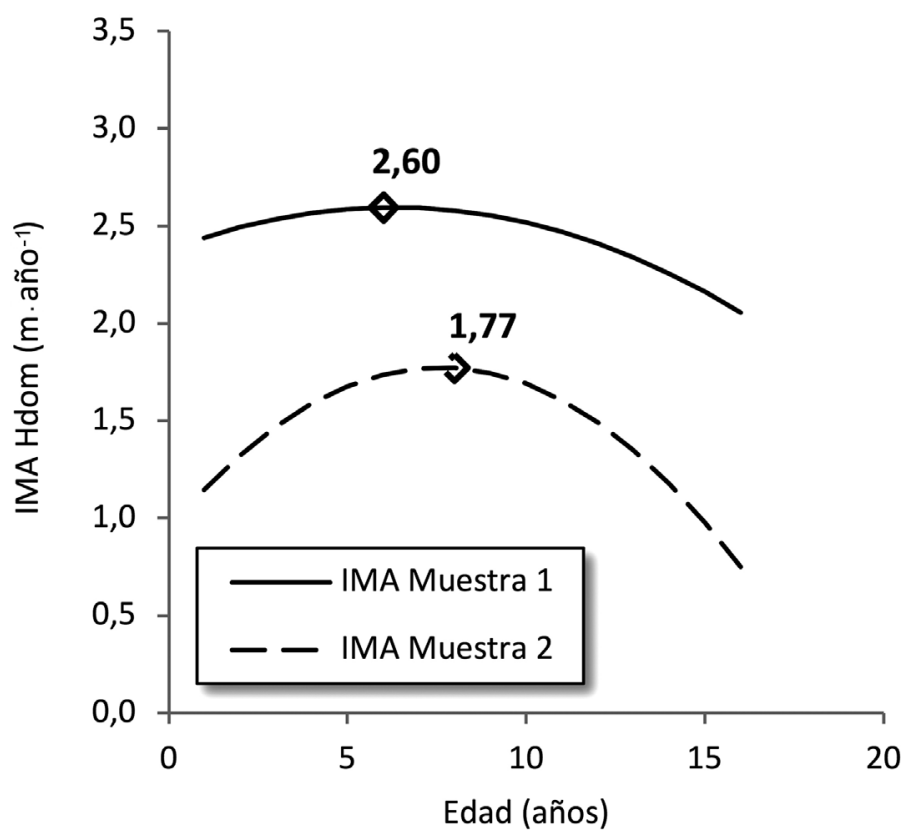

Fig. 4. Relación IMA (Hdom)-Edad para las muestras 1 y 2 de Vochysia guatemalensis, región Huetar Norte y Huetar Caribe, Costa Rica.

Con estas curvas se pone en evidencia las diferencias atribuibles a la calidad de sitio. En el mejor de los sitios, la curva de la Muestra 1 arranca en una posición superior con $2,44 \mathrm{~m}$, alcanzando un IMAmáx de 2,60 $\mathrm{m}$ a la edad de 6 años. La curva de la Muestra 2 inició y lamentablemente, con un IMA de 1,32 m, y solamente logró llegar a un IMAmáx de $1,77 \mathrm{~m}$ a la edad de 8 años.

El comportamiento de las curvas, de ICA (Figura 3) e IMA (Figura 4) de la Muestra 2 es típico de las condiciones de una calidad de sitio mala: a) arrancan lentamente, b) les toma más tiempo alcanzar su pico máximo, c) nunca alcanzan los niveles de las otra y d) presentan una declinación abrupta en ambos incrementos, al alcanzar valores muy bajos a edades tempranas. Todo esto viene a ratificar las discrepancias que se encontraron al graficar las alturas dominantes versus la edad, para ambas muestras, representadas en la Figura 2.

\section{Construcción de las curvas de índice de sitio} para la Muestra 1: modelo de Bailey y Clutter

Al correr el modelo de Bailey y Clutter con los datos de la Muestra 1, se obtuvieron los siguientes resultados: el valor de $\mathbf{b}$ es igual a $-5,18103$ (la pendiente de este modelo siempre va a ser negativa); por su parte, a es igual a 5,47261 y se encuentra dentro del rango de 2 a 7 indicado por Alder (1980). El valor del parámetro ĉ corresponde a 0,34326 y se encuentra entre 0,2 y 2 que son los valores propuestos por (Alder 1980) para la mayoría de las especies.

La Ecuación (1) se utilizó para ajustar los datos de la Muestra 1; a continuación se presentan los parámetros calculados durante la regresión:

$$
\operatorname{Ln}(\text { Hdom })=5.47261-5.18103 *(1 / \mathrm{Edad})^{\wedge 0.34326}
$$

Al incorporar dichos parámetros a la ecuación, con la cual se construyeron las curvas de 
índice de sitio (Ecuación (6)), queda expresada de la siguiente manera:

$$
\mathrm{H}=\mathrm{e}^{\wedge 5.47261} *\left[\mathrm{IS} / \mathrm{e}^{\wedge 5.47261}\right]^{\wedge\{9 / \mathrm{Ei}\}^{\wedge} 0.34326}
$$

\section{Construcción de las curvas de índice de sitio para la Muestra 2: Modelo de Prodan}

Al correr el modelo de Prodan con los datos de la Muestra 2, se obtuvieron los siguientes coeficientes, $\mathbf{a}=0,465523 ; \mathbf{b}=0,538814 ;$ y $\mathbf{c}$ $=0,0183663$. Ahora bien, al sustituir estos valores en la Ecuación (9), se obtiene la siguiente expresión,

$$
Z=0,465523+0,538814 * E+0,0183663 * E^{2}
$$

Finalmente, al utilizar estos coeficientes y sustituyendo el valor de $\mathbf{b}_{\mathbf{i}}$ en la Ecuación (12), se obtuvo la siguiente función, con la cual se construyó un sistema de curvas para la Muestra 2.

$$
\begin{gathered}
\mathrm{H}=\mathrm{E}_{\mathrm{i}}^{2} /(0,465523+\{(9 / \mathrm{IS})-(0,465523 / 9) \\
\left.-(0,0183663 * 9)\} * \mathrm{E}_{\mathrm{i}}+\left(0,0183663 * \mathrm{E}_{\mathrm{i}}^{2}\right)\right)
\end{gathered}
$$

Con las Ecuaciones (14) y (16) se logró generar 2 conjuntos de curvas polimórficas de índice de sitio, las cuales cubren todo el intervalo de edades y de alturas dominantes incorporadas en los datos de ambas muestras.

En el Cuadro 4 se presentan los estadísticos obtenidos como resultado de ajustar ambos modelos a los datos que conforman la Muestra 1 y la Muestra 2. En términos generales, los resultados de los análisis estadísticos son bastante buenos, por lo que se indica que los modelos escogidos resultaron ser apropiados en ambos casos.

Cuadro 4. Resumen de los estadísticos que respaldan ambas ecuaciones ajustadas mediante regresión lineal, a partir de los modelos de Bailey y Clutter y Prodan, para V. guatemalensis en las regiones Huetar Norte y Huetar Caribe de Costa Rica.

\begin{tabular}{clccccccc}
\hline $\begin{array}{c}\text { Condición } \\
\text { de Sitio }\end{array}$ & \multicolumn{1}{c}{ Modelo } & g.l. total & $\mathrm{r}$ & $\mathrm{R}^{2}$ & $\mathrm{R}^{2}$ adj & MSE & SEE & MAE \\
\hline Muestra 1 & $\begin{array}{l}\text { Bailey y Clutter } \\
(1974)\end{array}$ & 310 & $-0,94$ & 0,89 & 0,89 & 0,0565 & 0,2378 & 0,1699 \\
Muestra 2 & $\begin{array}{l}\text { Prodan } \\
(1951)\end{array}$ & 87 & 0,93 & 0,87 & 0,87 & 0,3886 & 0,6234 & 0,5269 \\
\hline
\end{tabular}

Nota: $r$ = coeficiente de correlación; $R^{2}$ = coeficiente de determinación; $R^{2}$ adj = coeficiente de determinación ajustado; MSE = cuadrado medio del error; SEE = error estándar de las estimaciones; y, MAE = error medio absoluto.

\section{Curvas de índice de sitio diferenciadas según los patrones de crecimiento observados}

Dadas las diferencias irreconciliables, encontradas en los patrones de crecimiento de la altura dominante representada en la Figura 2, se desarrollaron 2 juegos de curvas independientes con el fin de construir un sistema de clasificación de terrenos basado en los índices de sitio para V. guatemalensis. Para lograr esto, se construyeron 3 curvas de índice de sitio para las clases de crecimiento superior Muestra 1 y 3 curvas de índice de sitio para la clase de crecimiento inferior Muestra 2. Los índices de sitio, o índices de clasificación de sitios, fueron definidos utilizando la edad de 9 años como edad de referencia. 
Se observó (Figura 5), que las localidades de mejor crecimiento están representadas por los índices de sitio 19, 24 y 30; mientras que el grupo de parcelas que se encontraban creciendo en los peores sitios, fueron representadas por los índices de sitio 10, 14 y 18. Se puede ver en la figura anterior que a la edad de 9 años la curva de IS $=18$, que corresponde a la Muestra 2, se encuentra a muy poca distancia de la curva de IS $=19$ que pertenece a la Muestra 1. Luego, estas curvas se irán separando conforme aumenta la edad, hasta el punto de que ambos patrones de crecimiento se vuelven incompatibles.

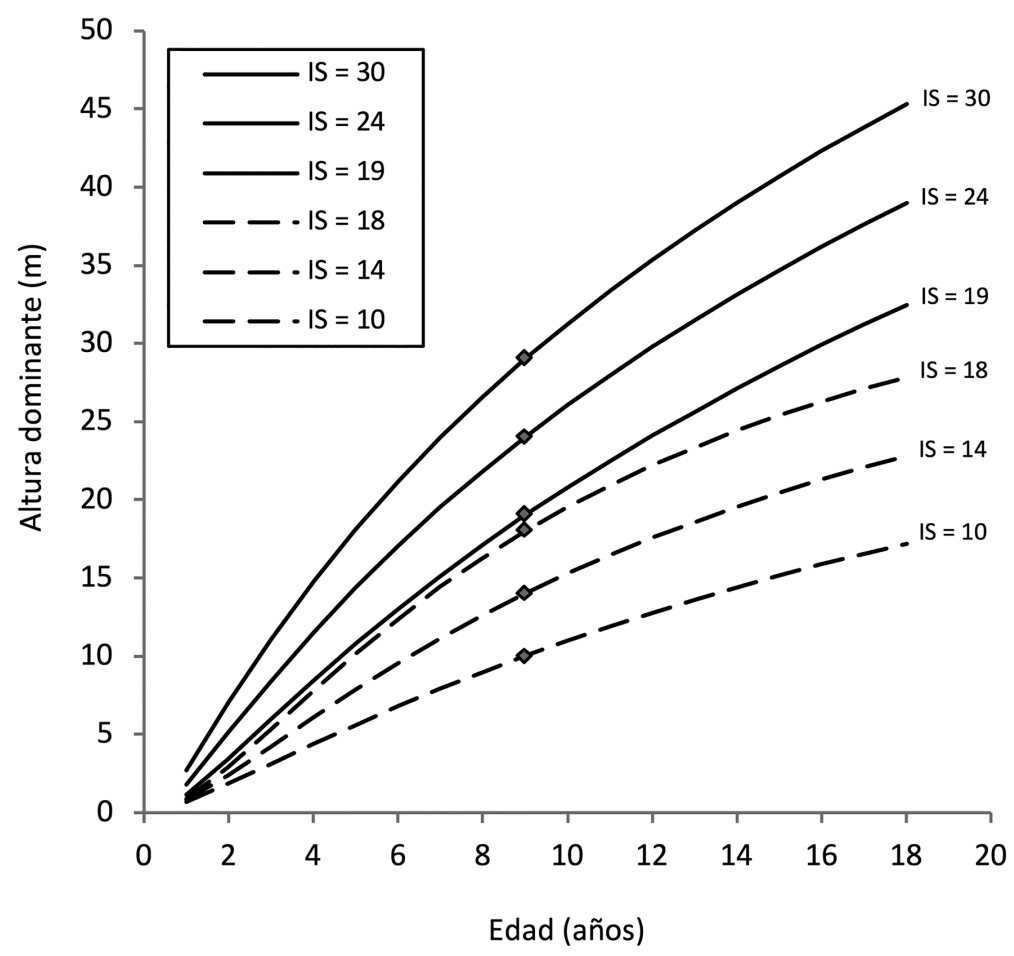

Fig. 5. Curvas de índice de sitio diferenciadas para las 2 condiciones observadas en las plantaciones de V. guatemalensis, en las regiones Huetar Norte y Huetar Caribe de Costa Rica.

Estas diferencias en el crecimiento pudieron deberse a factores edáficos que afectan el desarrollo de la especie en los sitios de mala calidad (por ejemplo un $\mathrm{pH}$ alto y una alta concentración de bases), o bien, en el caso de los sitios buenos, debido a factores edáficos que favorecen su crecimiento (por ejemplo una acidez elevada y una alta concentración de hierro y aluminio), tal y como lo establecieron (Mora et al. 2015, Camacho et al. 2016, Camacho et al. 2017).

\section{CONCLUSIONES}

Al analizar el desempeño de la altura dominante en relación con la edad, para $V$. guatemalensis en las regiones Huetar Norte y Huetar Caribe de Costa Rica, se pudo detectar la existencia de 2 patrones de crecimiento claramente diferenciados, lo cual permitió definir 2 zonas de crecimiento en el gráfico altura dominante-edad. 
Los sitios correspondientes a la Muestra 1 se pueden considerar de muy buena calidad. El crecimiento de las plantaciones en estos sitios es de muy bueno a excelente. Por otro lado, los sitios correspondientes a la Muestra 2 se pueden considerar de calidad de mala a muy mala para dicha especie.

La prueba de Levene indicó, con un 95\% de confianza, que todos los datos provienen de una misma población, mientras que la prueba $\mathrm{t}$ confirmó que existen diferencias significativas en los promedios de las alturas dominantes de ambas muestras.

Los gráficos de $\mathrm{ICA}_{\mathrm{Hdom}}$ e IMA $\mathrm{IMdom}_{\mathrm{H}}$ versus Edad corroboran las diferencias encontradas al aplicar las pruebas basadas en estadística inferencial. Ambos gráficos demostraron la superioridad de las plantaciones de la Muestra 1 sobre los datos de la Muestra 2, debido a que las plantaciones de esta última crecen de muy mala manera.

Las curvas de índice de sitio se construyeron al respetar las discrepancias encontradas en la muestra y para eso se utilizó una edad base de 9 años. Las localidades de mejor crecimiento están representadas por los índices de sitio 19, 24 y 30 ; mientras que las de peor crecimiento son representadas por los índices de sitio 10, 14 y 18 .

Las 2 ecuaciones presentaron un ajuste bastante aceptable, la ecuación para las Clases I y II (Bailey y Clutter) presentó los siguientes estadísticos: $\mathrm{r}=-0,94 ; \mathrm{R}^{2}$ ajust $=0,89 ; \mathrm{MSE}=0,0565 ; \mathrm{SEE}$ $=0,2378$; y la ecuación de la Clase III (Prodan): $\mathrm{r}=$ 0,$93 ; \mathrm{R}^{2}$ ajust $=0,87 ; \mathrm{MSE}=0,3886 ; \mathrm{SEE}=0,6234$.

\section{RECOMENDACIONES}

Se recomienda establecer la especie solamente en los índices de sitio 19 a 30, ya que bajo estas condiciones los árboles muestran su mejor desarrollo.

Es conveniente extender el muestreo a otras áreas y otras regiones donde se encuentra creciendo la especie, de manera que se pueda diversificar la información y aumentar el tamaño de la muestra.
Con el sistema de clasificación de sitios propuesto en este trabajo, se recomienda hacer acopio de toda la información relacionada con los diferentes tipos de suelo sobre los cuales se encuentra creciendo la especie, para establecer correlaciones entre las características físicas y químicas de los mismos con el crecimiento en altura dominante de $V$.guatemalensis.

\section{LITERATURA CITADA}

Asociación Costarricense para el Estudio de Especies Forestales Nativas (ACEN). 1992. Especie: Vochysia guatemalensis. In Memoria Segundo Encuentro Regional sobre Especies Forestales Nativas de la Zona Norte y Atlántica de Costa Rica. Heredia, Costa Rica. 87 p.

Alder D. 1980. Forest Volume Estimation and Yield Prediction. Vol. 2: Yield Prediction. FAO Forestry Paper 22/2, Rome, Italia. 194 p.

Andrade M. 2002. Evaluación del crecimiento de ocho especies forestales nativas en la Universidad EARTH, las Mercedes de Guácimo, Costa Rica. Universidad EARTH. 67 p.

Arias, D., Calvo-, J., Richter, D., Dohrenbusch, A. 2011 Productivity, Aboveground Biomass, Nutrient Uptake and Carbon Content in Fast-Growing Tree Plantations of Native and Introduced Species in the Southern Region of Costa Rica. Biomass and Bioenergy 35(5):1779-1788. DOI: http://dx.doi. org/10.1016/j.biombioe.2011.01.009

Bailey, RL; Clutter, JL. 1974. Base-age invariant polymorphic site curves. Forest Science 20(2):155-159.

Barraza, D., Díaz, J. 1999. Clasificación preliminar de sitios para plantaciones con Hyeronima alchorneoides, Vochysia guatemalensis, Vochysia ferruginea, Virola koschnyi y Terminalia amazonia, en la zona Nor-atlántica de Costa Rica. Tesis Lic. Heredia, Costa Rica, Universidad Nacional. 90 p.

Camacho, ME., Alvarado A., Fernández-Moya J. 2016. Vochysia guatemalensis Donn. Smith, an alternative species for reforestation on acid tropical soils. New Forests 47:1-16.

Camacho, ME., Alvarado, A., Fernández-Moya, J. 2017. Accumulation and export of nutrients in harvested wood of Vochysia guatemalensis in small-scale forest plantations. Journal of Tropical Forest Science 29(1):105-113.

Curtis, R. 1964. A stem-analysis approach to site-index curves. Forest Science 10:241-256.

Davis, LS; Johnson, KN. 1987. Forest Management. USA, McGraw-Hill Higher Education, Series in Forest Resources. 816 p. 
Delgado A. 2002. Crecimiento de las plantaciones de especies nativas y su relación con la motivación de finqueros a reforestar en la región Huetar Norte de Costa Rica. Informe de práctica de especialidad. Instituto Tecnológico de Costa Rica. Escuela de Ingeniería Forestal, Cartago, Costa Rica. 127 p.

Levene, H. 1960. Contributions to Probability and Statistics: Essays in Honor of Harold Hotelling. In Olkin, I; et al. (eds.). Stanford, California, USA, Stanford University Press. p. 278-292.

Montagnini, F; Sancho, F. 1994. Net nitrogen mineralization in soils under six indigenous tree species, an abandoned pasture and a secondary forest in the Atlantic lowlands of Costa Rica. Plant and Soil 162:117-124.

Mora, F; Meza, V. 2003. Curvas de índice de sitio para Teca (Tectona grandis Linn.) en la Vertiente del Pacífico de Costa Rica. In Plantaciones de Teca (Tectona grandis): Posibilidades y perspectivas para su desarrollo. Seminario y Grupo de Discusión Virtual. (Disco compacto), 26 al 28 de Noviembre de 2003, Heredia, Costa Rica, 16 p.

Mora, F; Meza, V; Chinchilla O; Chaves E. 2014. Curvas polimórficas de índice de sitio para Cupressus lusitanica en la Región Central de Costa Rica. Revista Forestal Venezolana 58:7-26.

Mora, F; Muñoz, R; Meza, V; Fonseca, W. 2015. Factores edáficos que influyen en el crecimiento de Vochysia guatemalensis en la región Huetar Norte de Costa Rica. Agronomía Costarricense 39(1):71-89.

Pérez, J. 1993. Efecto de la incorporación de hojarasca de especies forestales acumuladoras y no acumuladoras de aluminio sobre la distribución y las propiedades químicas de un suelo del trópico húmedo de Costa Rica. Tesis M.Sc. Costa Rica, CATIE/UCR. 193 p.

Prodan, M; Peters, R; Cox, F; Real, P. 1997. Mensura Forestal. IICA-GTZ. San José, Costa Rica. 561 p.

Richards, FJ. 1959. A flexible growth function for empirical use. J. Exp. Bot. 10:290-300.

Schumacher, FX. 1939. A new growth curve and its applications to timber yield studies. Journal of Forestry 37(11):819-820.

Sit, V; Poulin-Costello, M. 1994. Catalog of curves for curve fitting. Biometrics Information Handbook $\mathrm{N}^{\mathrm{o}}$. 4. Forest Science Research Branch, Ministry of Forests, Province of British Columbia, Victoria, B.C. $110 \mathrm{p}$.

Solís, M; Moya, R. 2004. Vochysia guatemalensis en Costa Rica. Fondo Nacional de Financiamiento Forestal. Proyecto Reforesta. Banco Mundial (en línea). Consultado mayo 2017. Disponible en http://www. fonafifo.com

Thompson, R; Alfaro R; Manning G. 1984. Site index curves for Lodgepole pine from southeastern Yukon. Environment Canada, Canadian Forestry Service, Information Report BC-X-247, Victoria, BC, Canada, Pacific Forest Research Center. 12 p. 